\title{
Nitramine Derivatives of NTO - A DFT Study
}

\section{Lemi Türker}

Department of Chemistry, Middle East Technical University, Üniversiteler, Eskişehir Yolu No: 1, 06800 Çankaya/Ankara, Turkey; e-mail: lturker@gmail.com; lturker@metu.edu.tr

\begin{abstract}
NTO is a nitrotriazole type explosive having good thermal stability and low chemical sensitivity. It is relatively insensitive to impact and shock. In the present study, nitramine derivatives of NTO are considered within the constraints of density functional theory at the level of B3LYP/6-31+G(d). The stabilities, some geometrical, physicochemical, and some molecular orbital characteristics etc., of the structures are considered. The calculated IR and UV-VIS spectra are presented and discussed.
\end{abstract}

\section{Introduction}

In modern ordnance, explosives having good thermal stability, impact and shock insensitivity and better performance are strongly desirable requirements. For these purposes the syntheses of nitrotriazoles as energetic materials and as intermediates to energetic materials have received a great deal of attention in the past decades [1]. NTO 1, (5-nitro-2,4-dihydro-3H-1,2,4-triazol-3-one) is the most extensively studied nitrotriazole explosives because it possesses good thermal stability [2], low chemical sensitivity to radiation damage [3] and is relatively insensitive to impact and shock [4]. The first report about NTO appeared in 1905 [5] however was erroneously reported as its hydroxy tautomer [6]. The explosive nature of it was recognized later. Various articles on NTO and its tautomers $\mathbf{2}$ and $\mathbf{3}$ have been published [7] and NTO was characterized as an insensitive energetic material in 1988 [8].

Received: January 13, 2019; Accepted: January 27, 2019

Keywords and phrases: NTO, explosives, nitramine, DFT, molecular orbital calculations, spectra.

Copyright (C) 2019 Lemi Türker. This is an open access article distributed under the Creative Commons Attribution License, which permits unrestricted use, distribution, and reproduction in any medium, provided the original work is properly cited. 


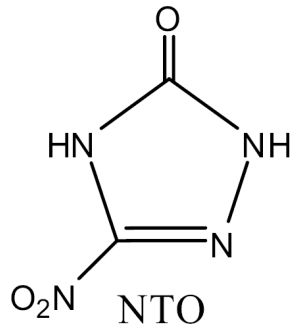

1

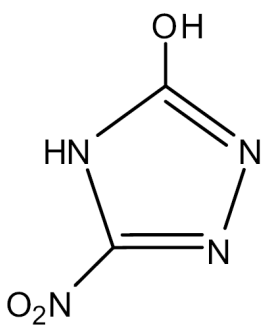

2

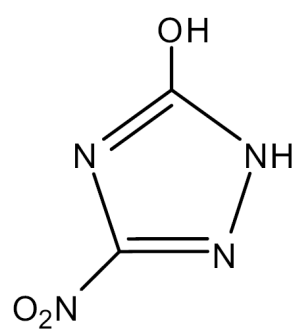

3

NTO was developed as an explosive compound in 1983 at Los Alamos center [8]. Its explosive performance characteristics are similar to RDX, but it has been observed that NTO is less sensitive [9]. Some applications of NTO have been reported, such as AFX644, which is a mixture of TNT:NTO:wax:Al (powder), having the proportion of 30:40:10:20. The baseline AFX-644 meets the United Nations' (UN) criteria for extremely insensitive detonating substances [10]. On the other hand, the charges containing NTO in the ratio of 25:25:50, RDX:NTO:TNT, improves significantly the sensitivity, mechanical properties and structural integrity of the explosive [11]. Shells charged with NTO, in the ratio of 40:60 NTO:TNT, are relatively insensitive to bullet impact and fuel fire tests [11]. Also, crystal forms of NTO have been studied and two polymorphic phases were discovered to exit [12]; the most stable $\alpha$-polymorph (for which an accurate crystal structure has yet to be determined because it undergoes a significant degree of twinning [13]) and the $\beta$-form which was reported to be unstable [14]. The structural aspects, chemical and explosives properties and thermal behavior of NTO, have been published $[15,16]$. On the other hand, the synthesis and structural characterization of metal salts of NTO, i.e., $\mathrm{K}, \mathrm{Cu}$ and Pb-NTO were also studied in the literature [17]. Many articles about NTO have piled in the literature [18-25], however there has been very rare information about the constitutional isomers or tautomers of NTO (4 and 5) [27-28].

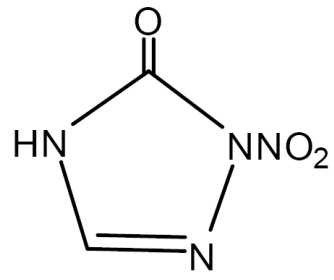

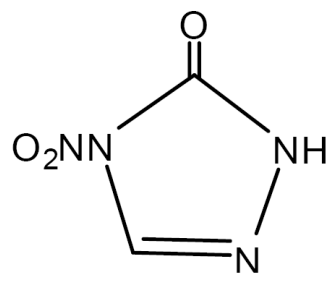

5 
In the present study, nitramine derivatives of NTO are considered within the constraints of density functional theory (DFT). Note that $\mathbf{4}$ and $\mathbf{5}$ are nitramines of 2,4dihydro-3H-1,2,4-triazol-3-one.

\section{Method of Calculations}

The initial geometry optimizations of all the structures leading to energy minima were achieved by using MM2 method followed by semi-empirical PM3 self-consistent fields molecular orbital (SCF MO) method [29, 30] at the restricted level [31]. Then, the structure optimizations were achieved within the framework of Haretree-Fock (HF) and finally by using density functional theory (DFT, B3LYP) [32, 33] at the level of B3LYP/6-31+G(d) (restricted closed-shell). Note that the exchange term of B3LYP consists of hybrid Hartree-Fock and local spin density (LSD) exchange functions with Becke's gradient correlation to LSD exchange [34]. The correlation term of B3LYP consists of the Vosko, Wilk, Nusair (VWN3) local correlation functional [35] and Lee, Yang, Parr (LYP) correlation correction functional. The normal mode analysis for each structure yielded no imaginary frequencies for the $3 N-6$ vibrational degrees of freedom, where $N$ is the number of atoms in the system. This indicates that the structure of each molecule corresponds to at least a local minimum on the potential energy surface. Furthermore, all the bond lengths were thoroughly searched in order to find out whether any bond cleavage occurred or not during the geometry optimization process. All these computations were performed by using SPARTAN 06 [36].

\section{Results and Discussion}

Figure 1 shows the optimized structures of the nitramines derived from NTO. Note that in 6-8 the $\pi$-connectivity of the ring is the same as NTO. The dinitro derivatives 6 and $\mathbf{7}$ are isomers of each other. The trinitro derivative $\mathbf{8}$ is also considered. The ring $\pi$-topologies of $\mathbf{9}$ and $\mathbf{1 0}$ are mutually the same but different than NTO. They might be considered as nitramines of $\mathbf{1 1}$ which is a 1,3-tautomer of NTO. Also note that $\mathbf{9}$ is isomeric with 6 and 7 all having the empirical formula of $\mathrm{C}_{2} \mathrm{HN}_{5} \mathrm{O}_{5}$.

Directions of dipole moment of the structures changes as well as their magnitudes as seen in Figure 1 and Table 1 which also displays some other properties of the structures considered. Note that the polarizability values, thus the $\log \mathrm{P}$ values are highly dependent on the number as well as the position of the nitro groups. Also note that dipole moments 


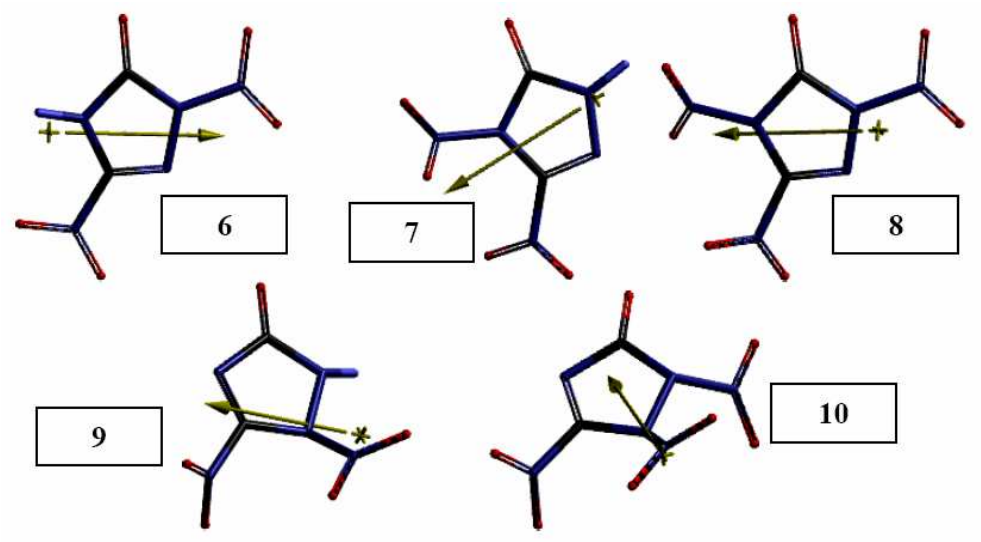

Figure 1. Optimized structures of the nitramines considered.
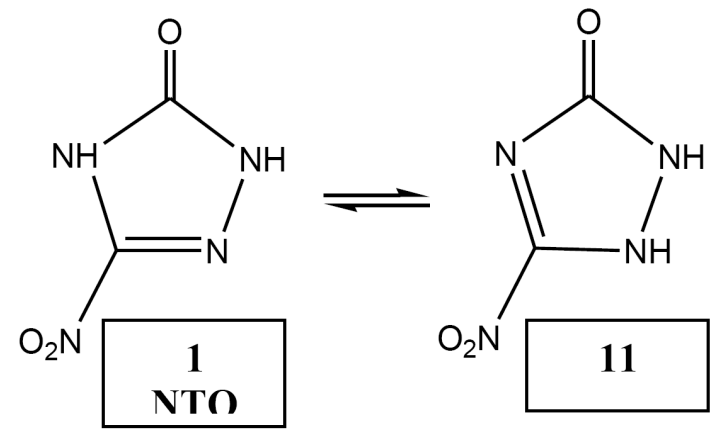

Table 1. Some properties of the structures considered.

\begin{tabular}{lrrrrr}
\hline \multicolumn{6}{c}{ Structures } \\
\hline Property & \multicolumn{1}{c}{$\mathbf{6}$} & $\mathbf{7}$ & $\mathbf{8}$ & \multicolumn{1}{c}{$\mathbf{9}$} & \multicolumn{1}{c}{$\mathbf{1 0}$} \\
\hline Formula & $\mathrm{C}_{2} \mathrm{HN}_{5} \mathrm{O}_{5}$ & $\mathrm{C}_{2} \mathrm{HN}_{5} \mathrm{O}_{5}$ & $\mathrm{C}_{2} \mathrm{~N}_{6} \mathrm{O}_{7}$ & $\mathrm{C}_{2} \mathrm{HN}_{5} \mathrm{O}_{5}$ & $\mathrm{C}_{2} \mathrm{~N}_{6} \mathrm{O}_{7}$ \\
Molecular weight & 175.06 & 175.06 & 220.06 & 175.06 & 220.06 \\
Dipole moment (Debye) & 2.60 & 3.92 & 0.19 & 3.11 & 0.93 \\
Area $\left(\AA^{2}\right)$ & 152.90 & 151.61 & 180.61 & 152.89 & 181.81 \\
Volume $\left(\AA^{3}\right)$ & 119.06 & 118.99 & 142.51 & 119.43 & 143.15 \\
Ovality & 1.31 & 1.30 & 1.37 & 1.30 & 1.37 \\
Polarizability & 50.04 & 49.98 & 51.85 & 49.99 & 51.83 \\
Log P & 0.58 & 0.58 & 1.14 & 0.97 & 1.53 \\
\hline
\end{tabular}


of isomeric structures $\mathbf{6}$ and $\mathbf{7}$ are highly different. In the case of $\mathbf{8}$ it is almost nil. Figure 2 shows the electrostatic potential (ESP) charges. Note that the ESP charges are obtained by the program based on a numerical method that generates charges that reproduce the electrostatic potential field from the entire wave function [36]. Positional variation of the nitro group in isomeric structures $\mathbf{6}$ and $\mathbf{7}$ highly affects the charges on all the atoms of the pentagonal ring backbone.
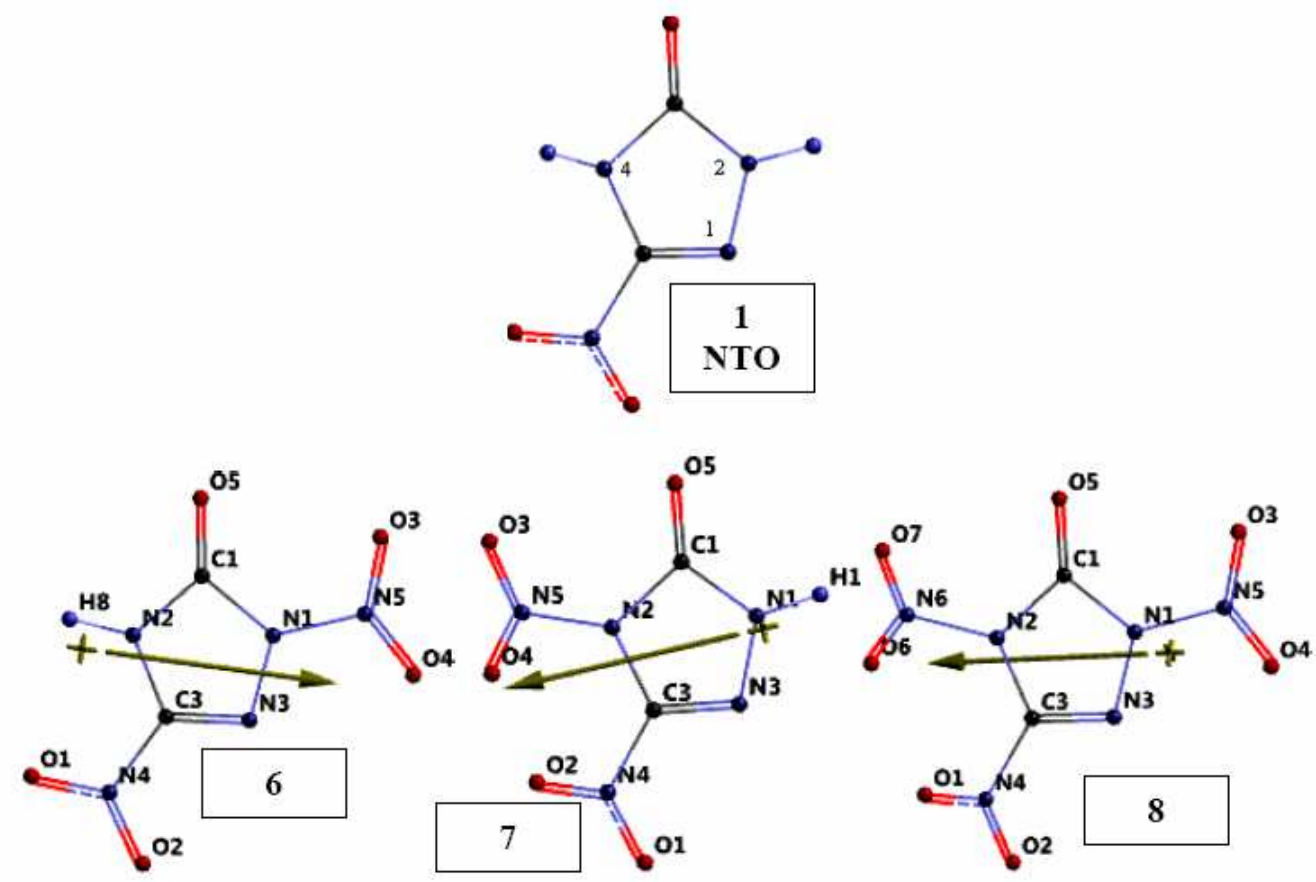

Figure 2. Charges (ESP) on the nitramines considered.

Figure 3 displays the bond lengths of the nitramines considered. The nitramine bond lengths (and carbonyl bond length in each case) are very comparable with each other, irrespective of position of the nitro groups.

The numbering of atoms of NTO and structures 6-8 are shown in Figure 4. The Mulliken and Löwdin bond orders for the structures having the same ring topology are listed in Tables 2 and 3, respectively. 

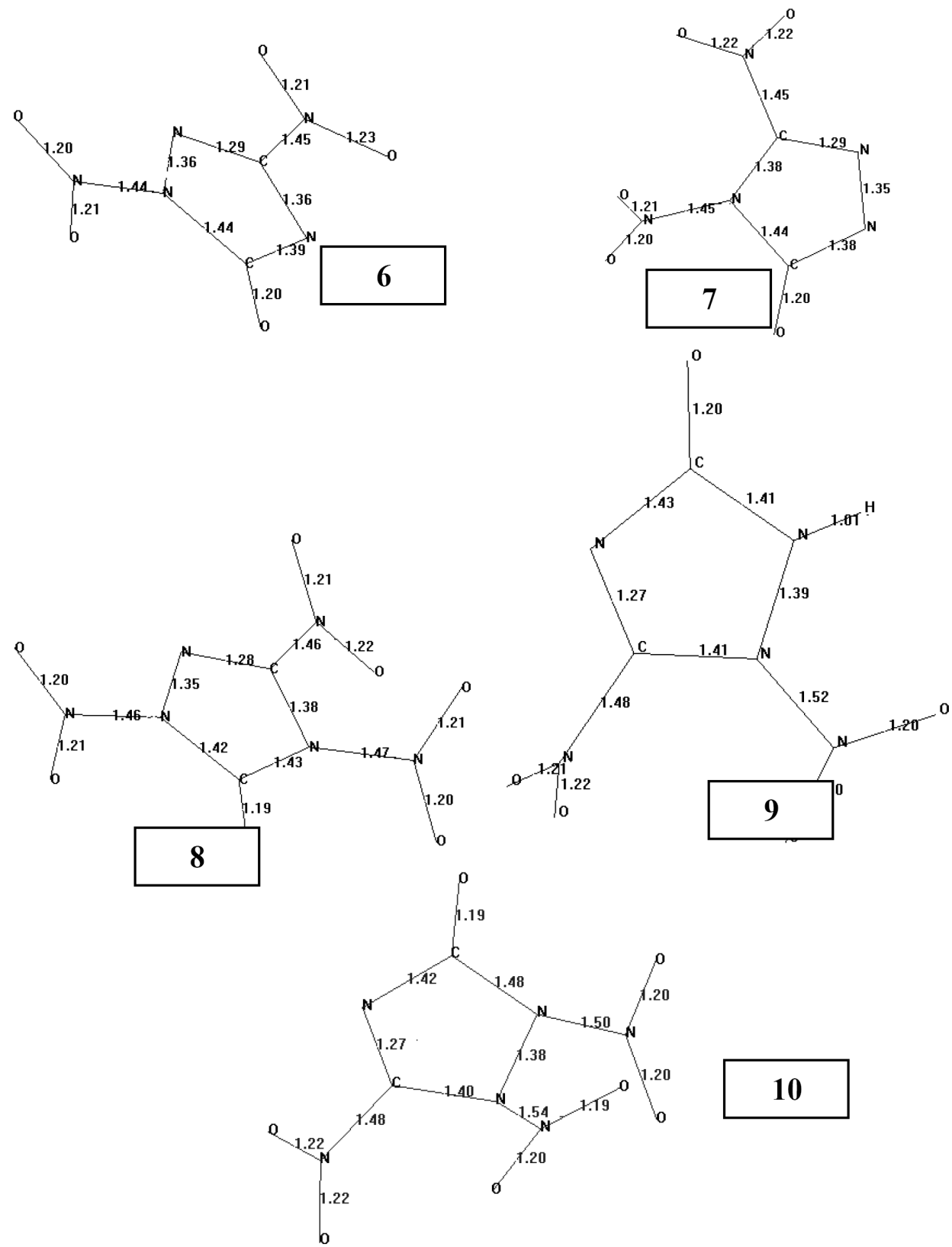

Figure 3. Bond lengths of the structures considered. 

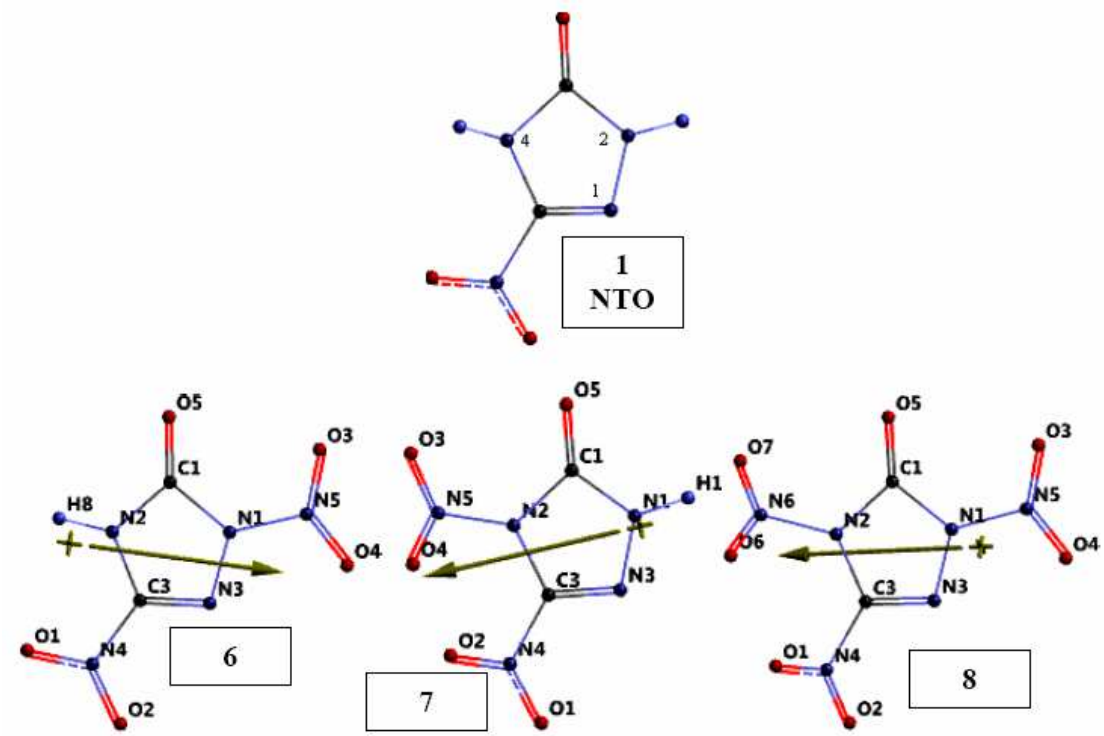

Figure 4. Numbering of atoms in NTO and structures 6-8.

Table 2. Mulliken bond orders of the structures 6-8.

\begin{tabular}{ccccccccc}
\hline & \multicolumn{3}{c}{ Structure-6 } & \multicolumn{3}{c}{ Structure-7 } & \multicolumn{3}{c}{ Structure-8 } \\
\hline Bond & Order & Remark & Bond & Order & Remark & Bond & Order & Remark \\
\hline C1 N2 & 1.017 & single & C1 N2 & 0.903 & single & C1 N2 & 0.908 & single \\
C1 N1 & 0.965 & single & C1 N1 & 1.074 & single & C1 N1 & 1 & single \\
C1 O5 & 1.565 & deloc & C1 O5 & 1.622 & deloc & C1 O5 & 1.667 & deloc \\
N2 C3 & 1.036 & single & N2 C3 & 1.037 & single & N2 C3 & 1.034 & single \\
N2 H8 & 0.699 & sing-H & N2 N5 & 0.782 & single & N2 N6 & 0.598 & single \\
C3 N3 & 1.82 & double & C3 N3 & 1.77 & double & C3 N3 & 1.864 & double \\
C3 N4 & 0.706 & single & C3 N4 & 0.734 & single & C3 N4 & 0.731 & single \\
N3 N1 & 1.173 & single & N3 N1 & 1.368 & deloc & N3 N1 & 1.274 & deloc \\
N1 N5 & 1.001 & single & N1 H1 & 0.708 & sing-H & N3 N5 & 0.607 & single \\
N4 O1 & 1.895 & double & N4 O1 & 2.117 & double & N4 O1 & 2.055 & double \\
N4 O2 & 2.175 & double & N4 O2 & 2.002 & double & N4 O2 & 2.153 & double \\
N5 O3 & 1.903 & double & N5 O3 & 1.886 & double & N5 O3 & 1.959 & double \\
N5 O4 & 1.826 & double & N5 O4 & 1.933 & double & N5 O4 & 1.858 & double \\
& & & & & & N6 O6 & 1.975 & double \\
& & & & & & N6 O7 & 1.914 & double \\
\hline
\end{tabular}


Table 3. Lowdin bond orders of the structures 6-8.

\begin{tabular}{|c|c|c|c|c|c|c|c|c|}
\hline \multicolumn{3}{|c|}{ Structure-6 } & \multicolumn{3}{|c|}{ Structure-7 } & \multicolumn{3}{|c|}{ Structure-8 } \\
\hline Bond & Order & Remark & Bond & Order & Remark & Bond & Order & Remark \\
\hline $\mathrm{C} 1 \mathrm{~N} 2$ & 1.197 & single & $\mathrm{C} 1 \mathrm{~N} 2$ & 1.045 & single & $\mathrm{C} 1 \mathrm{~N} 2$ & 1.057 & single \\
\hline C1 N1 & 1.038 & single & $\mathrm{C} 1 \mathrm{~N} 1$ & 1.219 & single & C1 N1 & 1.066 & single \\
\hline C1 O5 & 2.157 & double & $\mathrm{C} 1 \mathrm{O} 5$ & 2.137 & double & $\mathrm{C} 1 \mathrm{O} 5$ & 2.187 & double \\
\hline $\mathrm{N} 2 \mathrm{C} 3$ & 1.258 & deloc & $\mathrm{N} 2 \mathrm{C} 3$ & 1.15 & single & N2 C3 & 1.154 & single \\
\hline $\mathrm{N} 2 \mathrm{H} 8$ & 0.738 & sing-H & N2 N5 & 0.974 & single & N2 N6 & 0.942 & single \\
\hline $\mathrm{C} 3 \mathrm{~N} 3$ & 1.766 & double & $\mathrm{C} 3 \mathrm{~N} 3$ & 1.802 & double & $\mathrm{C} 3 \mathrm{~N} 3$ & 1.797 & double \\
\hline C3 N4 & 0.94 & single & C3 N4 & 0.931 & single & C3 N4 & 0.911 & single \\
\hline N3 N1 & 1.353 & deloc & N3 N1 & 1.425 & deloc & N3 N1 & 1.34 & deloc \\
\hline N1 N5 & 0.986 & single & N1 H1 & 0.75 & sing-H & N1 N5 & 0.956 & single \\
\hline N4 O1 & 1.961 & double & $\mathrm{N} 4 \mathrm{O} 1$ & 2.016 & double & $\mathrm{N} 4 \mathrm{O} 1$ & 1.979 & double \\
\hline $\mathrm{N} 4 \mathrm{O} 2$ & 2.028 & double & $\mathrm{N} 4 \mathrm{O} 2$ & 1.977 & double & $\mathrm{N} 4 \mathrm{O} 2$ & 2.032 & double \\
\hline $\mathrm{O} 1 \mathrm{O} 2$ & 0.461 & single & $\mathrm{O} 1 \mathrm{O} 2$ & 0.462 & single] & $\mathrm{O} 1 \mathrm{O} 2$ & 0.467 & single \\
\hline N5 O3 & 2.022 & double & N5 O3 & 2.051 & double & N5 O3 & 2.032 & double \\
\hline \multirow[t]{3}{*}{ N5 O4 } & 2.02 & double & N5 O4 & 2.009 & double & N5 O4 & 2.034 & double \\
\hline & & & & & & N6 O6 & 2.012 & double \\
\hline & & & & & & N6 O7 & 2.068 & double \\
\hline
\end{tabular}

Although Mulliken population analysis has some deficiencies, it is very popular. Large positive overlaps between basis functions on different atoms are indicative of an existing chemical bond. Löwdin population analysis is often less used because of its computational requirements. In Tables 2 and 3 some of the bond orders are greater than 2.00 which stand for some of the nitro groups. Note that both the Mulliken and Löwdin results are basis set dependent [37].

Table 4 shows the total electronic energy (E), zeropoint vibrational energy (ZPE) and the corrected total electronic energy $\left(\mathrm{E}_{\mathrm{c}}\right)$. The data in the table indicate that of the isomeric structures $\mathbf{6 , 7}$ and $\mathbf{9}$, structure-6 is more stable than $\mathbf{7}$ and $\mathbf{9}$. It means that in structures-6 and 7 positions 2 and 4 of NTO (see Figure 4 for numbering of the atoms) are not equivalent in terms of contributions to the stability. Structurally those positions are obviously different but electronically $\mathrm{N}(2)$ stabilizes whereas $\mathrm{N}(4)$ destabilizes the system. The construction of nitramine bond at those positions decreases the electronic density of nitrogen- 2 or -4 because of the electron attracting effect of nitro group. The 
Table 4. Some energies of the structures considered.

\begin{tabular}{cccc}
\hline Structure & $\mathbf{E}$ & $\mathbf{Z P E}$ & $\mathbf{E}_{\mathbf{c}}$ \\
\hline $\mathbf{6}$ & -1907318.24 & 176.61 & -1907141.63 \\
$\mathbf{7}$ & -1907286.91 & 176.96 & -1907109.95 \\
$\mathbf{8}$ & -2444084.76 & 178.69 & -2443906.07 \\
$\mathbf{9}$ & -1907246.98 & 175.166183 & -1907071.81 \\
$\mathbf{1 0}$ & -2444057.78 & 176.791654 & -2443880.98 \\
\hline
\end{tabular}

Energies in $\mathrm{kJ} / \mathrm{mol}$.

following resonance structures can be written for NTO. The canonical strucure-12 should have destabilizing effect on the resonance hybrid because it has negatively charged nitrogen (N(1)) adjacent to the other nitrogen atom. Thus lone-pair-anion destabilization prevails in structure-12. However, if a nitro group is substituted on N(4), the contribution of resonance structure $\mathbf{1 2}$ considerably decreases. Thus its destabilizing effect substantially diminishes. Note that $\pi$-topology of $\mathbf{9}$ is different than $\mathbf{1}$ (NTO), $\mathbf{6}$ and $\mathbf{7}$.
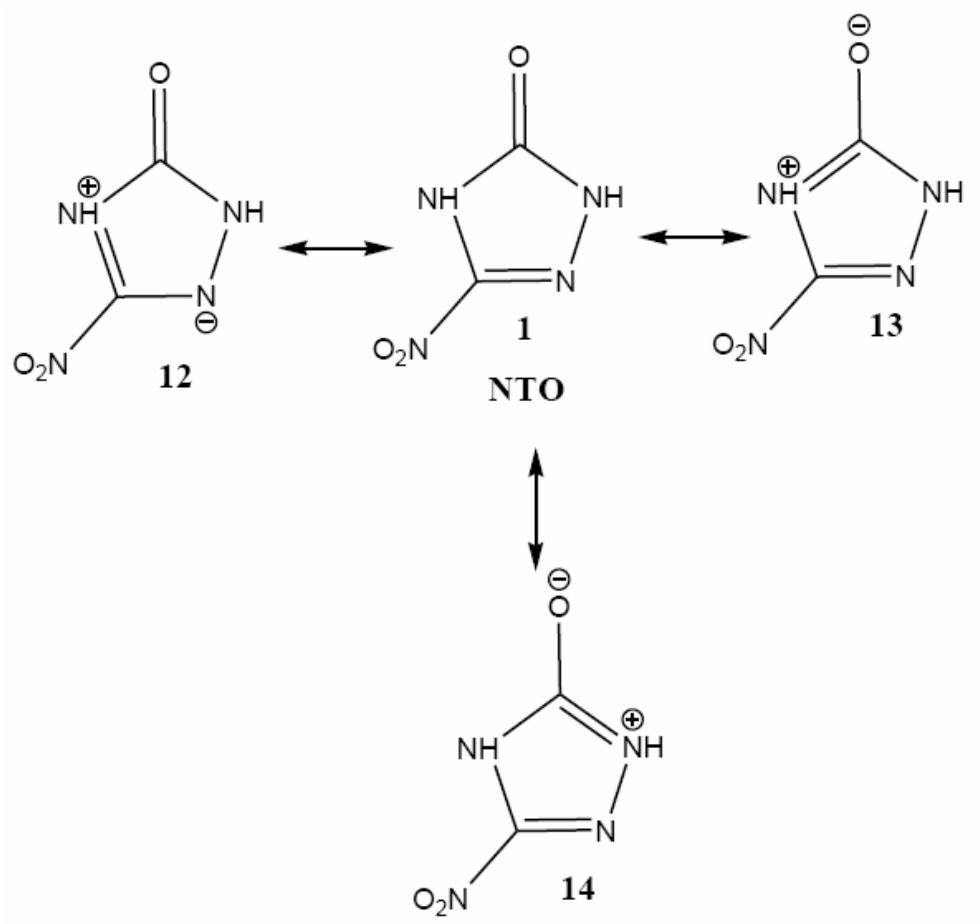
On the other hand, in between nitrogen atoms N(1) and N(2), it seems lone-pair-lonepair repulsive interaction occurring. However, due to the different hybridization character of those nitrogens, the effect should not reach any appreciable extent.

Figure 5 shows the calculated infrared spectra of the nitramines considered. Note that structures $\mathbf{6}$ and $\mathbf{7}$ are isomeric ones also having the same $\pi$-topology in the ring.
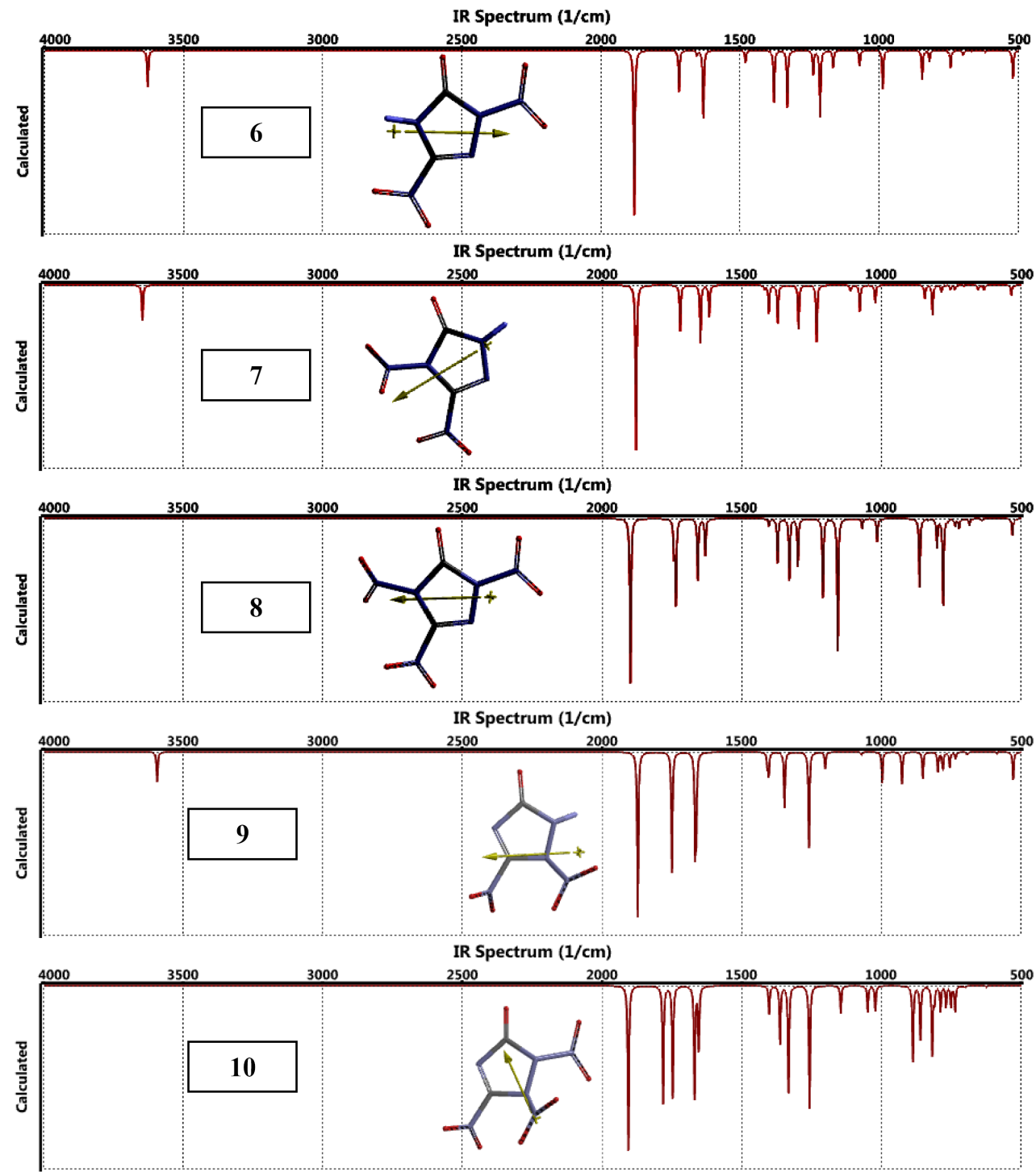

Figure 5. IR spectra of the nitramines considered. 
Their N-H stretching occurs at 3629 and $3645 \mathrm{~cm}^{-1}$, respectively. The carbonyl stretchings of 6-8 happen at 1880, 1878 and $1898 \mathrm{~cm}^{-1}$, respectively. The carbonyl stretchings of $\mathbf{9}$ and $\mathbf{1 0}$ also occur in that region. Asymmetric stretching of $\mathrm{N}=\mathrm{O}$ of the nitramine $\mathrm{NO}_{2}$ group in 6 occurs at $1719 \mathrm{~cm}^{-1}$. In 7 it happens at $1720 \mathrm{~cm}^{-1}$ and in 8 at 1735 and $1742 \mathrm{~cm}^{-1}$. Note that in $\mathbf{8}$ two nitramine moieties exist. The $\mathrm{N}=\mathrm{O}$ stretching of ordinary $\mathrm{NO}_{2}$ group of 6-8 occurs at 1632, 1647 and $1657 \mathrm{~cm}^{-1}$, respectively.

The calculated UV-VIS spectra of the presently considered structures are shown in Figure 6. They all absorb in the UV region. Although, 7 and 8 exhibit only one discernable absorption maximum, 6 possesses two (at $255.8 \mathrm{~nm}$ and $318.8 \mathrm{~nm}$ ). Note that in 6 the nitro groups are coplanar with the ring whereas in all the rest of the structures they have different degrees of twisting, thus not coplanar with the ring $\pi$-system. This situation should have generated various transition possibilities which could be responsible for the obtained calculated spectra.

Table 5 displays the main UV absorptions, their strengths and transition moment components. Table 6 displays the HOMO, LUMO energies and interfrontier molecular orbital energy gaps $(\Delta \boldsymbol{\varepsilon})$ of the structures considered. The order of HOMO energies is $\mathbf{1 0}<\mathbf{8}<\mathbf{9}<\mathbf{6}<\mathbf{7}$ whereas the LUMO energies follow the order of $\mathbf{1 0}<\mathbf{8}<\mathbf{6}<\mathbf{9}<\mathbf{7}$. Consequently, the order of interfrontier molecular orbital gaps becomes $\mathbf{6}<\mathbf{7}<\mathbf{9}<\mathbf{8}<\mathbf{1 0}$. 

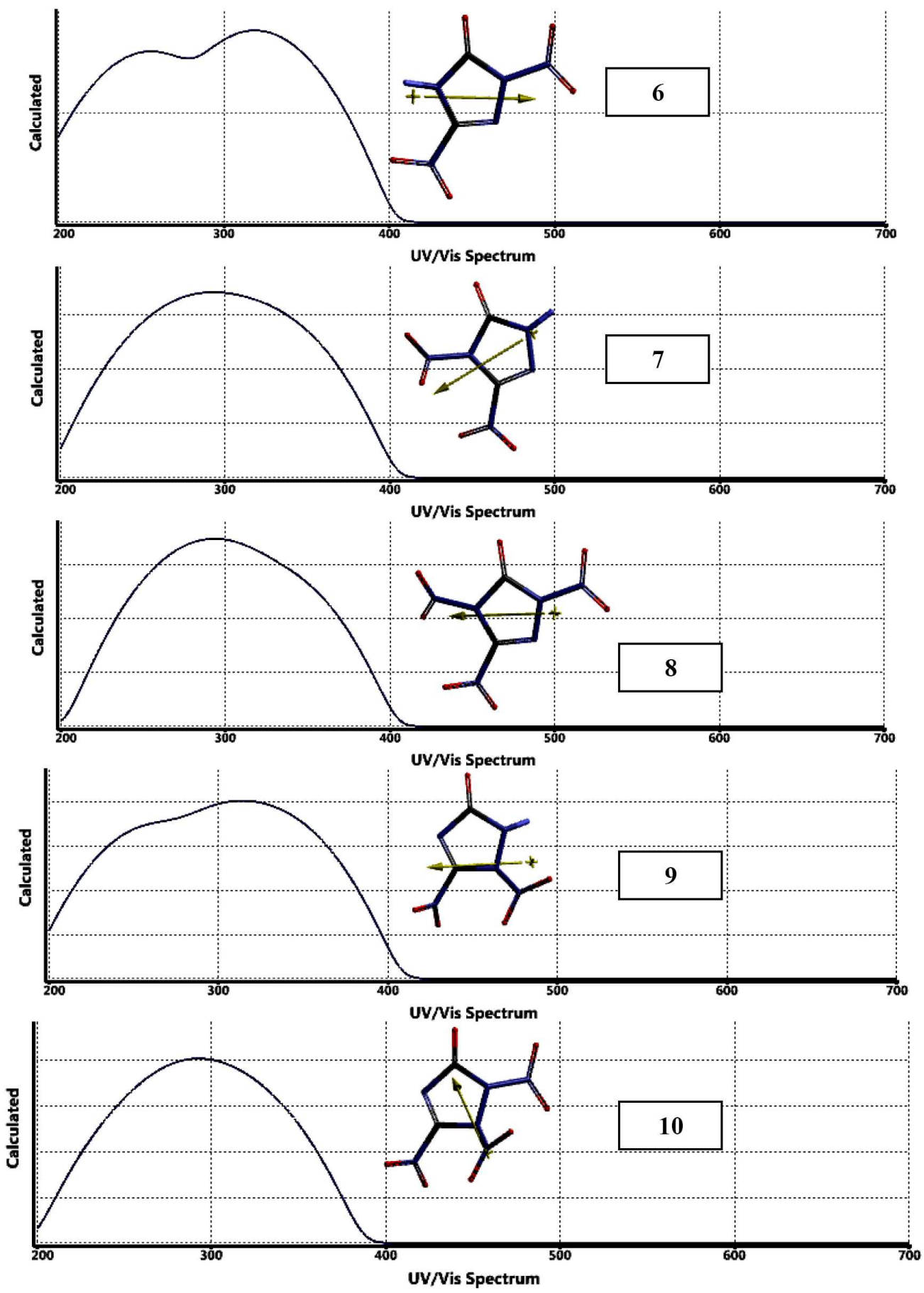

Figure 6. UV-VIS spectra of the nitramines considered. 
Table 5. Main absorptions, their strengths and transition moment components.

\section{Transition moment components}

\begin{tabular}{cccccc}
\hline Structure & $\boldsymbol{\lambda}_{\max }$ & Strength & $\mathbf{X}$ & $\mathbf{Y}$ & $\mathbf{Z}$ \\
\hline $\mathbf{6}$ & 318.87 & 0.1956 & 1.1308 & 0.0880 & 0.8756 \\
& 255.80 & 0.0515 & 0.5356 & -0.2878 & -0.2523 \\
\multirow{2}{*}{$\mathbf{*}$} & 308.29 & 0.0572 & 0.4474 & 0.4941 & -0.3687 \\
& 286.33 & 0.0753 & 0.6388 & 0.5442 & 0.0731 \\
$\mathbf{8}$ & 301.74 & 0.0615 & -0.7094 & 0.0070 & -0.3274 \\
& 293.13 & 0.0591 & 0.6562 & 0.0387 & 0.3721 \\
& 282.69 & 0.0541 & -0.4836 & -0.0291 & -0.5186 \\
$\mathbf{9}$ & 330.85 & 0.0235 & -0.4410 & -0.2362 & -0.0733 \\
& 318.87 & 0.0155 & 0.3261 & 0.2067 & 0.1177 \\
& 307.22 & 0.0409 & 0.6324 & -0.0012 & 0.1150 \\
$\mathbf{1 0}$ & 261.41 & 0.0133 & 0.3366 & 0.0232 & 0.0164 \\
& 311.70 & 0.0131 & -0.3528 & -0.0778 & -0.0603 \\
& 293.76 & 0.0432 & -0.3022 & -0.1909 & -0.5382 \\
& 285.50 & 0.0202 & -0.0181 & -0.1352 & -0.4141 \\
\hline
\end{tabular}

Table 6. The HOMO, LUMO energies and interfrontier molecular orbital energy gaps $(\Delta \boldsymbol{\varepsilon})$ of the structures considered.

\begin{tabular}{cccc}
\hline Structure & HOMO & LUMO & $\boldsymbol{\Delta} \boldsymbol{\varepsilon}$ \\
\hline $\mathbf{6}$ & -820.76 & -417.06 & 403.70 \\
$\mathbf{7}$ & -816.07 & -388.95 & 427.12 \\
$\mathbf{8}$ & -877.85 & -435.75 & 442.10 \\
$\mathbf{9}$ & -847.10 & -409.64 & 437.46 \\
$\mathbf{1 0}$ & -912.64 & -441.99 & 470.65 \\
\hline
\end{tabular}

Energies in $\mathrm{kJ} / \mathrm{mol}$.

Figure 7 shows some molecular orbital energies of the nitramines considered which have the same ring $\pi$-topology. Thus variations are due to positional and conformational changes of the substituents in $\mathbf{6}$ and 7. Structures $\mathbf{6}$ and $\mathbf{7}$ are positional isomers of each other and their HOMO and LUMO energies, especially the HOMO energies, do not greatly differ from each other, but the inner lying molecular orbital energy levels do. On the other hand, introducing the third nitro group to form another nitramine moiety 
substantially lowers the HOMO and LUMO energies in $\mathbf{8}$ as compared to others due to the electron withdrawing effect of the nitro group. Structure-9 is isomeric with $\mathbf{6}$ and $\mathbf{7}$ but $\mathbf{1 0}$ is different from 6-7 in many respects.
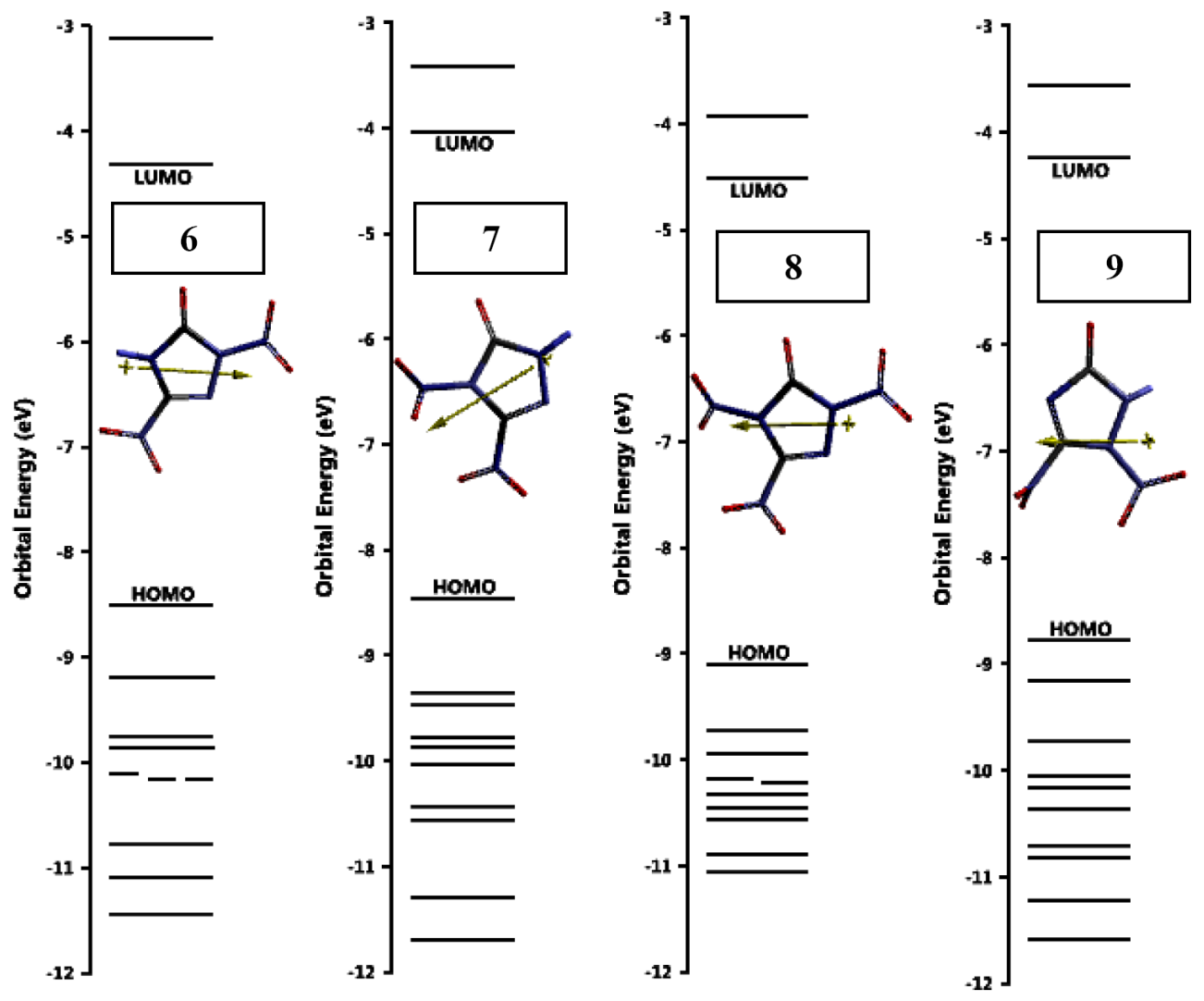

Figure 7. Some molecular orbital energies of the nitramines 6-9.

Figure 8 shows the HOMO and LUMO patterns of the nitramines considered. The orbitals generally have $\pi$-symmetry. In the case of HOMO the overlap between N1C1N2 centers (see Figure 4 for numbering of the atoms) is appreciable. The contribution of carbonyl group into the LUMO is nil in structures 6 and 8.

Figure 9 shows the electrostatic potential maps of the nitramine considered. Generally all the structures are characterized with positive potential field in the core of the structures and varying degrees of negative potential field exists around the periphery of the structures. 


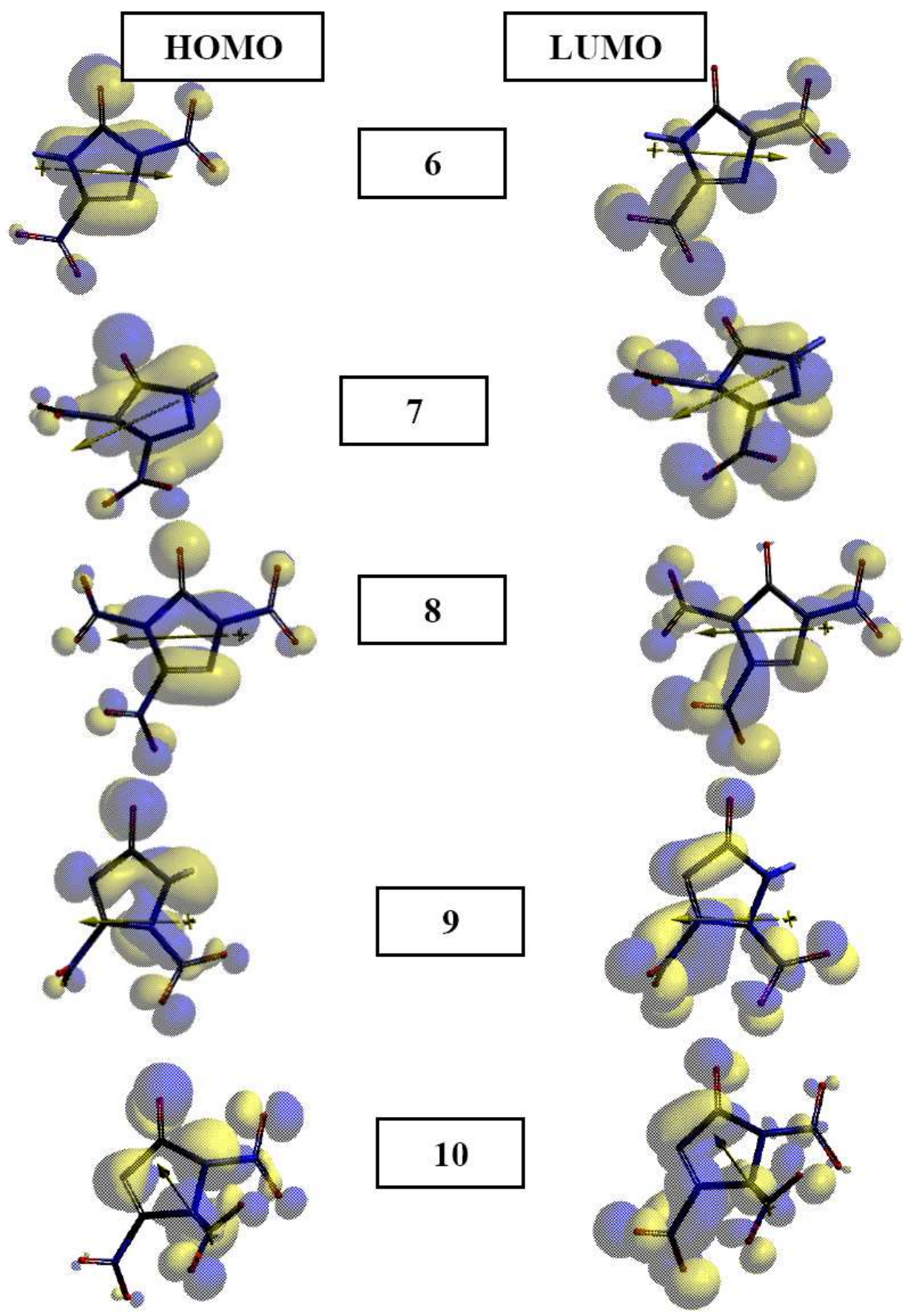

Figure 8. The HOMO and LUMO patterns of the nitramines considered. 

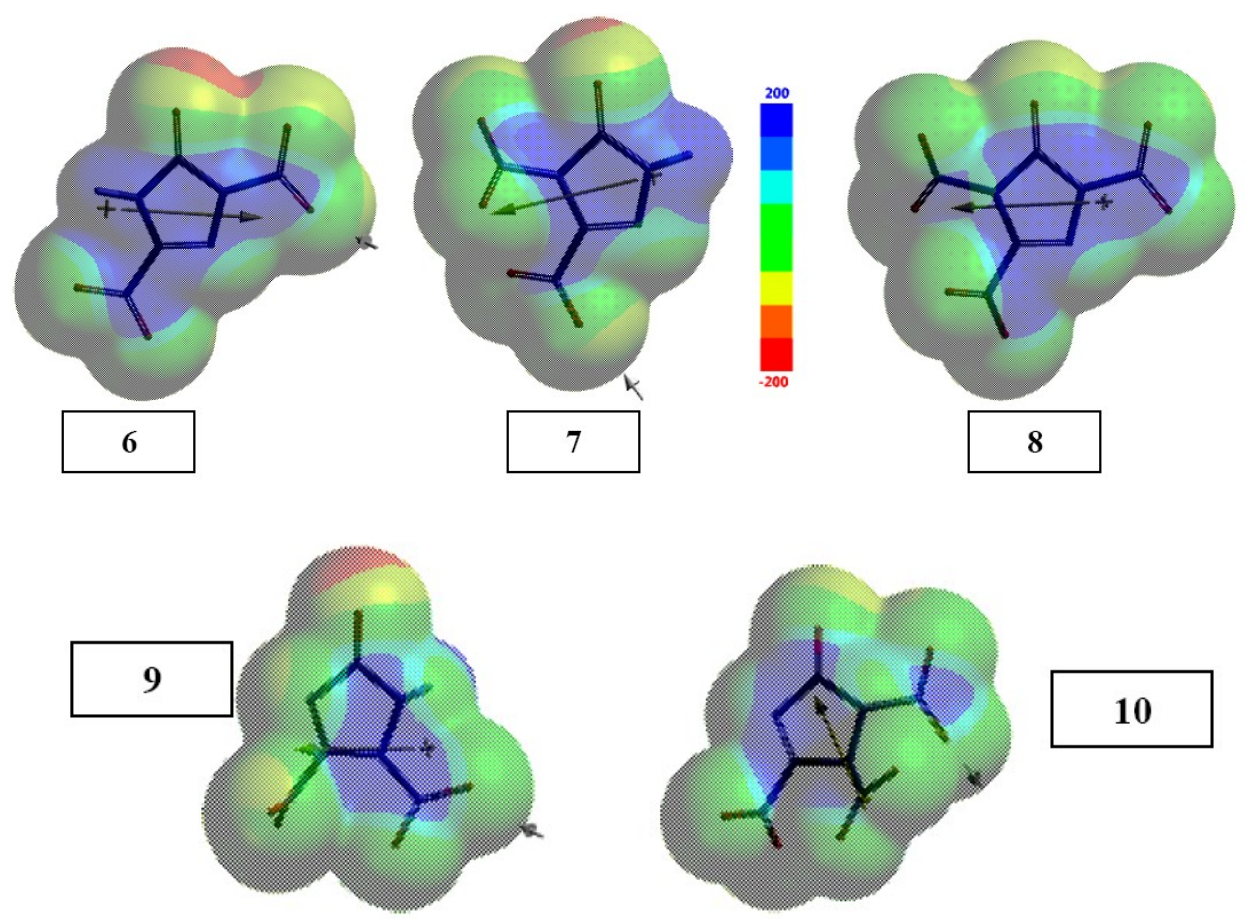

\section{0}

Figure 9. Electrostatic potential maps of the nitramine considered.

\section{Conclusion}

Of the various nitramine derivatives of NTO presently considered, three of them $(\mathbf{6}$, 7 and 9) are isomeric and structure-6 is more stable than 7. Structure-9 is also isomeric with 6 and 7 but ring $\pi$-topology of it is different. Although IR spectra of them are very similar, the UV spectrum of structure-6 differs from 7 having two maxima in the UV region. Their HOMO energies are quite comparable but the LUMO energy of $\mathbf{7}$ is higher. Consequently the interfrontier molecular energy gaps follow the order of $\mathbf{6}<\mathbf{7}<\mathbf{9}<\mathbf{8}<\mathbf{1 0}$. The results in the present study are dictated mainly by the lack of symmetry present in the ring system and location and conformation of the substituents. If the syntheses of these nitramines are managed, their ballistic properties might be worth investigated.

\section{References}

[1] O. Yuxiang, C. Boren, L. Jiarong, D. Shuan, L. Jianjuan and J. Huiping, Synthesis of nitro derivatives of triazoles, Heterocycles 38 (1994), 1651-1664. 
[2] E. F. Rothgery, D. E. Audette, R. C. Wedlich and D. A. Csejka, The study of the thermal decomposition of 3-nitro-1,2,4-triazol-5-one (NTO) by DSC, TGA-MS, and accelerating rate calorimetry (ARC), Thermochim. Acta 185(2) (1991), 235-243.

[3] B. C. Beard and J. Sharma, Early decomposition chemistry of NTO (3-nitro-1,2,4-triazol5-one), J. Energ. Mater. 11(4-5) (1993), 325-343.

[4] Y. Xie, R. Hu, X. Wang, X. Fu and C. Zhunhua, Thermal behavior of 3-nitro-1,2,4triazol-5-one and its salts, Thermochim. Acta 189 (1991), 283-296.

[5] V. W. Manchot and R. Noll, Triazole derivatives, Justus Liebigs Ann. Chem. 343 (1905) $1-27$.

[6] C. F. Kroger, R. Mietchen, H. Fank, M. Siemer and S. Pilz, 1,2,4-Triazoles. XVII. Nitration and bromination of 1,2,4-triazolinones, Chem. Ber. 102 (1969), 755-766.

[7] Y. M. Wang, C. Chen and S. T. Lin, Theoretical studies of the NTO unimolecular decomposition, J. Mol. Struct. (Theochem) 460 (1999), 79-102.

[8] K. Y. Lee and M. D. Coburn, 3-nitro-1,2,4-triazol-5-one, a less sensitive explosive, Report No. LA-10302-MS; Order No. DE86009787, 1985, 7 pp.

[9] D. C. Sorescu, T. R. L. Sutton, D. L. Thompson, D. Beardallm and C. A. Wight, Theoretical and experimental studies of the structure and vibrational spectra of NTO, $J$. Mol. Struct. 384 (1996), 87-99.

[10] B. M. Kosowski and R. C. Taylor, New processing aid and emulsifier for energeics, Int. Annu. Conf. ICT (27th) (1996) 152.1-152.11.

[11] F. C. Fouche, V. Schalkwyk and C. Gideon, TNT-based insensitive munitions, Int. Annu. Conf. ICT (27th) (1996) 69.1-69.12.

[12] E. A. Zuhorova and A. A. Pinkerton, Chemical bonding in energetic materials: $\beta$-NTO, Acta Crystallogr. Sect. B Struct. Sci. B57 (2001), 359-365.

[13] N. Bolotina, K. Krischbaum and A. A. Pinkerton, Energetic materials: $\alpha$-NTO crystallizes as a four-component triclinic twin, Acta Crystallogr. B61 (2005), 577-584.

[14] K. Y. Lee and R. Gilardi, NTO polymorphs, in: Proc. Materials Research Soc. Symp., vol. 296, Structure and Properties of Energetic Materials, 1993, pp. 237-242.

[15] R. J. Spear, C. N. Louey and M. G. Wolfson, A Preliminary Assessment of NTO as an Insensitive High Explosive, MRL-TR-89-18 Materials Research Laboratory, Vic., Australia, 1989.

[16] V. L. Zbarskii, V. V. Kuz'min and N. V. Yudin, Synthesis and properties of 1-nitro-4,5dihydro-1H-1,2,4-triazol-5-one, Russian J. Chem. 40 (2004), 1069-1070. 
[17] Y. Xie, R. Z. Hu, X. Y. Wang and X. Y. Fu, Thermal behavior of NTO and its salts, in: Proceedings of the 17th International Pyrotech Seminar (Combined with the Second Beijing International Symposium on Pyrotechnics and Explosives), vol. 1, Beijing Institute of Technology Press, Beijing, 1991, pp. 509-512.

[18] P. B. Kulkarni, T. S. Reddy, J. K. Nair, A. N. Nazare, M. B. Talawar, T. Mukundan and S. N. Asthana, Studies on salts of 3-nitro-1,2,4-triazol-5-one (NTO) and 2,4,6trinitroanilino benzoic acid (TABA): potential energetic ballistic modifiers, J. Hazard Mater. 123(1-3) (2005), 54-60.

[19] F. J. Owens, Calculation of energy barriers for bond rupture in some energetic molecules, J. Mol. Struct. (Theochem) 370(1) (1996), 11-16.

[20] K. Y. Lee, L. B. Chapman and M. D. Coburn, 3-Nitro-1,2,4-triazol-5-one, a less sensitive explosive, J. Energ. Mater. 5 (1987), 27-33.

[21] Y. Kohno, O. Takahashi and K. Saito, Theoretical study of initial decomposition process of NTO dimer, Phys. Chem. Chem. Phys. 3(14) (2001), 2742-2746.

[22] R. I. Hiyoshi, Y. Kohno and J. Nakamura, Vibrational assignment of energetic material 5nitro-2,4-dihydro-1,2,4-triazol-3-one (NTO) with labeled isomers, J. Phys. Chem. A 108(27) (2004), 5915-5920.

[23] H. M. Ma, J. R. Song, W. Dong, R. Z. Hu, G. H. Zhai and Z. Y. Wen, Molecular structure, the quantum chemical investigation and the thermal behavior of the dimethylamine salt of 3-nitro-1,2,4-triazol-5-one, $\left(\mathrm{CH}_{3}\right)_{2} \mathrm{NH}_{2}{ }^{+} \mathrm{C}_{2} \mathrm{~N}_{4} \mathrm{O}_{3} \mathrm{H}^{-}$, J. Mol. Struct. (Theochem) 678(1-3) (2004), 217-222.

[24] G. Singh and S. P. Felix, Studies on energetic compounds. Part 32. Crystal structure, thermolysis and applications of NTO and its salts, J. Mol. Struct. 649(1-2) (2003), 71-83.

[25] J. Vagenknecht, P. Marecek and W. A. Trzcinski, Sensitivity and performance properties of TEX explosives, J. Energ. Mater. 20(3) (2003), 245-253.

[26] L. Türker and T. Atalar, Quantum chemical study on 5-nitro-2,4-dihydro-3H-1,2,4triazol-3-one (NTO) and some of its constitutional isomers, J. Hazard Mat. A 137 (2006), $1333-1344$.

[27] V. L. Zbarskii, V. V. Kuz'min and N. V. Yudin, Synthesis and properties of 1-nitro-4,5dihydro-1H-1,2,4-triazol-5-one, Russ. J. Org. Chem. 40(7) (2004), 1069-1070.

[28] C. Meredith, T. P. Russell, R. C. Mowrey and J. R. McDonald, Decomposition of 5-nitro2,4-dihydro-3H-1,2,4-triazol-3-one (NTO): energetics associated with several proposed initial routes, J. Phys. Chem. A 102 (1998), 471-477. 
[29] J. J. P. Stewart, Optimization of parameters for semi empirical methods I, Method. J. Comput. Chem. 10 (1989), 209-220.

[30] J. J. P. Stewart, Optimization of parameters for semi empirical methods II, Appl. J. Comput. Chem. 10 (1989), 221-264.

[31] A. R. Leach, Molecular Modeling, Essex: Longman, 1997.

[32] W. Kohn and L. J. Sham, Self-consistent equations including exchange and correlation effects, Phys. Rev. 140 (1965), 1133-1138.

[33] R. G. Parr and W. Yang, Density Functional Theory of Atoms and Molecules, London: Oxford University Press, 1989.

[34] A. D. Becke, Density-functional exchange-energy approximation with correct asymptotic behavior, Phys. Rev. A 38 (1988), 3098-3100.

[35] S. H. Vosko, L. Vilk and M. Nusair, Accurate spin-dependent electron liquid correlation energies for local spin density calculations: a critical analysis, Can. J. Phys. 58 (1980), 1200-1211.

[36] SPARTAN 06, Wavefunction Inc., Irvine CA, USA, 2006.

[37] D. C. Young, Computational Chemistry, New York: Wiley, 2001. 